\title{
Mechanical Flexibility in 1D Coordination Polymer Crystals
}

\section{Biswajit Bhattacharya, Adam A. L. Michalchuk and Franziska Emmerling}

BAM Federal Institute for Materials Research and Testing, Richard-Willstätter-Strasse 11, 12489 Berlin (Germany)

\author{
biswajit.bhattacharya@bam.de
}

Mechanical flexibility in single crystals of covalently bound materials is a fascinating and poorly understood phenomenon. [1-2] We present here the first example of a plastically flexible one-dimensional (1D) coordination polymer (CP). The compound $[\mathrm{Zn}(\mu-$ Cl)2(3,5-Cl2Py)2]n (1), is flexible over two crystallographic faces.[3] Through the combination of microscopy, diffraction, and spectroscopic studies we probe the structural response of the crystal lattice to mechanical bending. Our results suggest that mechanical bending occurs by displacement of the coordination polymer chains. Based on experimental and theoretical evidence, we propose a new model for mechanical flexibility in 1D coordination polymers. [4] To understand the role of weak interactions on mechanical flexibility of CP crystals, we explored a family of metal halide-based CPs isomorphous with 1, based on combination of two different metals $(\mathrm{Zn}$ and $\mathrm{Cd})$ and two halogens $(\mathrm{Cl}$ and $\mathrm{Br})$. We demonstrate how these simple modifications can tune the mechanical flexibilities across a significant range from plastic to delaminating, and ultimately to elastic. We rationalized these remarkable changes of mechanical properties by ab initio simulations.

[1] Saha, S., Mishra, M. K., Reddy, C. M. \& Desiraju G. R. (2018). Acc. Chem. Res. 51, 2957-2967.

[2] Đaković, M., Borovina, M., Pisačić, M., Aakeröy, C. B., Soldin, Ž., Kukovec, B. M. \& Kodrin, I. (2018). Angew. Chem. Int. Ed. 57, 1480114805 .

[3] Bhattacharya, B., Michalchuk, A. A. L., Silbernagl, D., Rautenberg, M., Schmid, T., Feiler, T., Reimann, K., Ghalgaoui, A., Sturm, H., Paulus, B. \& Emmerling, F. (2020). Angew. Chem. Int. Ed. 59, 5557-5561.

[4] X. Liu, AAL Michalchuk, B Bhattacharya, F Emmerling, and CR Pulham (2021) Nat. Commun. $12,3871$.

Keywords: Mechanical flexibility; Coordination polymer; ab initio simulations 